\title{
Diabetes Melito Transitório Relacionado à Terapia Com L-Asparaginase
}

\begin{abstract}
RESUMO
$\mathrm{O}$ tratamento de neoplasias hematológicas malignas com L-asparaginase tem sido associado com o desenvolvimento de diabetes melito (DM) em cerca de $1-2 \%$ dos casos. A associação com corticosteróides tem efeito sinérgico na ocorrência dessa complicação. Este artigo relata o desenvolvimento de DM transitório em uma adolescente de 13 anos, tratada com L-asparaginase e dexametasona devido a leucemia linfoblástica aguda. O DM foi diagnosticado 120 dias após o início da terapia com L-asparaginase, requerendo o uso de insulina por 12 meses. Investigação para auto-imunidade contra células beta pancreáticas foi negativa. Também não foram encontradas alterações laboratoriais sugestivas de pancreatite. $\mathrm{O} D \mathrm{DM}$ associado à L-asparaginase é insulinopênico e de caráter transitório, desaparecendo após a suspensão do medicamento. O diagnóstico do DM associado à L-asparaginase é baseado na associação temporal com o uso da droga e na exclusão de outras causas. Não existe um teste laboratorial capaz de diagnosticá-lo. Portanto, a investigação para DM tipo 1A, DM tipo 1B, DM induzido por corticoterapia e DM secundário a pancreatite tóxica é fundamental. $O$ tratamento com insulinoterapia deve ser acompanhado de perto, uma vez que este tipo de diabetes tem um curso transitório. (Arq Bras Endocrinol Metab 2007;51/4:635-638)
\end{abstract}

Descritores: L-asparaginase; Diabetes melito; Hiperglicemia

\section{ABSTRACT}

Transient Diabetes Mellitus Related to L-Asparaginase Therapy.

Treatment of hematological malignancies with L-asparaginase has been associated with diabetes mellitus in about $1-2 \%$ of patients. The concomitant use of steroids has an additional deleterious effect. In this article, we report the occurrence of diabetes in a 13 year-old girl treated with Lasparaginase and dexamethasone for acute lymphoblastic leukemia. The diabetes developed 120 days after the drug was started, requiring insulin therapy for 12 months. Anti-islet autoantibody was negative, and there were no laboratory findings suggestive of pancreatitis. The DM related do L-asparaginase therapy is insulinopenic and transient, resolving with suspension of the drug. The diagnosis of this type of diabetes is based on its temporal relationship with the L-asparaginase and in the exclusion of other known causes. There is no laboratory test capable of elucidating the diagnosis. Therefore, investigation to rule out type 1A DM, type 1B DM, insulin-resistant DM induced by corticotherapy and DM secondary to toxic pancreatitis is of utmost importance. The insulin therapy must be followed closely, since this is a transient form of diabetes. (Arq Bras Endocrinol Metab 2007;51/4:635-638)

Keywords: L-asparaginase; Diabetes mellitus; Hyperglycemia apresentação de caso

\author{
CRÉSIO Alves \\ Carina Chaves \\ MABEL SOUZA
}

Serviço de Endocrinologia

Pediátrica, Faculdade de

Medicina, Universidade Federal da Bahia, Salvador, BA.
Recebido em 19/12/06

Revisado em 26/01/07

Aceito em 23/02/07 
A ASPARAGINASE É UMA ENZIMA purificada a partir das bactérias Escherichia coli e Erwinia chrysanthemi, sendo responsável pela deaminação extracelular da asparagina, aminoácido essencial para a síntese protéica de algumas células tumorais deficientes na produção da enzima asparagina-sintetase $(1,2)$. Sua principal indicação tem sido no tratamento de neoplasias hematológicas, principalmente a leucemia linfoblástica aguda (LLA). Nessa situação, ela é usada como agente indutor de remissão da doença, uma vez que as células leucêmicas, por serem incapazes de sintetizar a asparagina, necessitam do aporte exógeno desse aminoácido $(1,3)$.

Diversos efeitos colaterais têm sido atribuídos a essa droga, como alterações neurológicas, gastrintestinais, hematológicas, hepáticas, pancreáticas, renais e metabólicas $(3,4)$. Hiperglicemia tem sido relatada em cerca de $1-2 \%$ dos pacientes tratados com L-asparaginase, principalmente quando se utiliza o preparado produzido a partir da Escherichia coli $(5,6)$. Apesar de mesma efetividade e menor toxicidade, o custo do tratamento com o preparado da Erwinia chrysanthemi é significativamente superior $(5,6)$.

Devido à raridade dessa complicação, este artigo tem por objetivo relatar um caso de diabetes melito (DM) transitório induzido pelo uso de asparaginase e discutir os possíveis mecanismos diabetogênicos a ela associados.

\section{RELATO DE CASO}

S.L.P., sexo feminino, 13 anos, IMC: $20,31 \mathrm{~kg} / \mathrm{m}^{2}$ (P.50-75), com diagnóstico de leucemia linfocítica aguda (LLA) realizado em 17/05/04 através de mielograma, iniciou quimioterapia em 24/05/04 pelo protocolo GBTLI-LLA93 - alto risco. Quatro meses após o início da quimioterapia com L-asparaginase derivada da Escherichia coli (10.000 UI/m²/dose), dexametasona $\left(6 \mathrm{mg} / \mathrm{m}^{2} /\right.$ dose, por 28 dias a cada dois meses) e vincristina $\left(1,4 \mathrm{mg} / \mathrm{m}^{2} / \mathrm{semana}\right)$, apresentou poliúria, polidipsia, polifagia, perda de peso $(9,7 \mathrm{~kg}$ em 5 dias) e elevação dos níveis glicêmicos, necessitando hospitalização para controle metabólico. À admissão, a menor, além de hiperglicemia, apresentava hálito cetótico, desidratação, cetonúria e acidose metabólica (gasometria arterial: $\mathrm{pH} \mathrm{7,2,}$ bicarbonato $8 \mathrm{mEq} / \mathrm{L}$, excesso de base $-12 \mathrm{mEq} / \mathrm{l}$ ). Após a obtenção do controle metabólico, a paciente teve alta hospitalar em uso de insulina humana NPH na dose de 20 UI, subcutânea, antes do café da manhã e 6 UI antes do jantar $(0,5 \mathrm{UI} / \mathrm{kg} / \mathrm{dia})$. Dosagens de anticorpos para auto-imunidade pancreática foram negativas ao diagnóstico, quatro meses depois de iniciada a insulinoterapia e cinco meses após a resolução do DM (tabela 1). Os níveis de insulina permaneceram dentro da faixa da normalidade, com níveis de peptídeo $\mathrm{C}$ abaixo dos valores de referência no início do quadro e normalização após resolução do DM (tabela 1). Não foram detectadas alterações em enzimas hepáticas ou pancreáticas, e a paciente não apresentou manifestações clínicas sugestivas de pancreatite.

Durante o acompanhamento ambulatorial, evoluiu com redução progressiva da necessidade de insulina, a qual foi suspensa 1 ano após ter sido iniciada. Entretanto, nos últimos seis meses antes da suspensão da insulina, a paciente não mais usava insulina NPH, e só administrava insulina regular, nas raras ocasiões em que

Tabela 1. Secreção insulínica, controle glicêmico e autoimunidade pancreática em paciente com diagnóstico de diabetes melito relacionado à terapia com L-asparaginase.

\begin{tabular}{lccc}
\hline $\begin{array}{l}\text { Avaliação } \\
\text { laboratorial }\end{array}$ & Diagnóstico do DM & $\begin{array}{c}\text { Cronologia dos exames } \\
\mathbf{4} \text { meses após o } \\
\text { diagnóstico do DM }\end{array}$ & $\begin{array}{c}\mathbf{5} \text { meses após } \\
\text { suspensão da insulina }\end{array}$ \\
$\begin{array}{l}\text { Glicemia em jejum } \\
\text { (VR: } 70-99 \mathrm{mg} / \mathrm{dL})\end{array}$ & 358 & 80 & 84 \\
$\begin{array}{l}\text { HbA1c } \\
\text { (VR: }<7 \%)\end{array}$ & 8,4 & $\mathrm{NR}$ & 4,9 \\
$\begin{array}{l}\text { Insulina } \\
\text { (<28 } \mu \mathrm{UI} / \mathrm{mL})\end{array}$ & 0,6 & 9,9 & $\mathrm{NR}$ \\
$\begin{array}{l}\text { Peptídeo-C } \\
\text { (VR: } 1,1-5 \mathrm{ng} / \mathrm{mL})\end{array}$ & 0,7 & 1,1 & 5,0 \\
$\begin{array}{l}\text { Anti-IA2 } \\
\text { (VR: }<0,5 \mathrm{Ul} / \mathrm{mL})\end{array}$ & 0,3 & 0,3 & 0,1 \\
$\begin{array}{l}\text { Anti-GAD } \\
\text { (VR: }<1 \mathrm{Ul} / \mathrm{mL})\end{array}$ & 0,2 & 0,3 & 1,0 \\
\hline Anti-GAD: & & & \\
\hline
\end{tabular}

Anti-GAD: anticorpo antidecarboxilase do ácido glutâmico, Anti-IA2: anticorpo antitirosina-fosfatase, DM: diabetes melito, HbA1C: hemoglobina glicada, NR: não realizado. 
a glicemia estava elevada. Hemoglobina glicada era de $8,4 \%$ ao diagnóstico ( $\mathrm{VR}<7 \%$ ) e $4,9 \%$ quando da suspensão da insulina. Avaliação de glicemias e hemoglobina glicada tem-se mantido normal no seguimento 15 meses após a interrupção da insulinoterapia.

\section{DISCUSSÃO}

Hiperglicemia tem sido relatada como complicação do uso da L-asparaginase há várias décadas $(7,8)$. Vários mecanismos têm sido propostos para explicar seu efeito diabetogênico: insulinopenia secundária à inibição da produção deste hormônio pela droga (9), hipoinsulinemia secundária à ocorrência de pancreatite $(4,10)$, alteração na fase precoce de liberação de insulina (10), redução no número de receptores de insulina (11) e redução na síntese de insulina como resultado da depleção da asparagina pela L-asparaginase, desde que este aminoácido é necessário para síntese da insulina $(12,13)$.

A associação de L-asparaginase com corticóides (incremento da gliconeogênese hepática e resistência insulínica) aumenta em dez vezes o risco dessa complicação $(6,13)$. O estudo de Bäckman sobre redução da ocorrência de diabetes melito pós-transplante renal após a diminuição ou eliminação o uso de corticóides oferece suporte a esses achados falando a favor de um efeito tóxico do corticóide sobre a célula beta pancreática (14). Mais recentemente, Duval e cols. (15) relataram menor prevalência de DM associado à terapia com Escherichia coli-asparaginase e Erwiniaasparaginase. Talvez o uso de prednisona, e não dexametasona, na fase de indução possa explicar a diferença de suas menores taxas de DM em relação a outros estudos $(0,6-1,4 \%$ vs. $1-10 \%)$.

O uso concomitante de drogas como metrotexate e vincristina não tem sido relacionado ao aumento no risco de DM (4). Alguns estudos mostraram presença de hiperglucagonemia similar a outros tipos de diabetes associados a insulinopenia $(7,9,12,13)$.

$\mathrm{O}$ efeito diabetogênico da L-asparaginase só ocorre numa minoria dos pacientes, sugerindo que o processo neoplásico, a dose e esquema terapêutico utilizado, e características inatas do hospedeiro participam, através de mecanismos ainda não conhecidos, da patogênese desse distúrbio (6). Além disso, a L-asparaginase derivada da Escherichia coli, usada por esta paciente, parece ser mais associada à toxicidade do que o preparado derivado da Erwinia chrysanthemi $(5,16)$.

No presente relato de caso, os seguintes achados sugerem o diagnóstico de diabetes melito insu- lino-dependente: apresentação inicial em cetoacidose diabética e níveis séricos de insulina e peptídeo-C inapropriadamente baixos para o estado hiperglicêmico. Isto confirma a natureza insulinopênica do DM relacionado à L-asparaginase, afastando a possibilidade de diabetes insulino-resistente induzido pelo uso da dexametasona. Em relação à etiologia do DM, embora o processo leucêmico pudesse tê-lo precipitado, a persistente negatividade dos auto-anticorpos para imunidade pancreática torna pouco provável a possibilidade do tipo lA ou auto-imune, uma vez que esses anticorpos estão presentes ao diagnóstico na maioria dos pacientes, especialmente o anti-GAD, cuja positividade pode persistir por até 10 anos (17). A ausência de sinais clínicos e laboratoriais de pancreatite exclui outra possível causa de diabetes. Embora alguns estudos tenham demonstrado hiperglucagonemia relativa similar a outros tipos de diabetes associados à insulinopenia $(12,13)$, a contribuição do aumento do glucagon no desenvolvimento da hiperglicemia ainda é questionável, podendo apenas representar um fenômeno secundário $(7,9,12,13)$. Na paciente relatada, os níveis de glucagon não foram dosados. O DMl tipo B, não auto-imune ou idiopático, cuja genética não é bem conhecida, poderia ter sido precipitado pelo estresse da leucemia, porém o prolongado período de remissão do DM, mais de um ano sem usar insulina, afasta essa a possibilidade. Assim, por exclusão de outras causas conhecidas de DM, pelo caráter transitório do mesmo e por sua associação temporal com o uso da L-asparaginase, foi estabelecido o diagnóstico de DM relacionado ao uso dessa droga.

Este tipo de DM tem caráter transitório, ocorrendo, na maioria dos casos, após a primeira semana do início da terapia com a L-asparaginase, e apresentando remissão após suspensão do medicamento (13). O tempo médio de uso de insulina, após início dos sintomas, variou, em alguns relatos, de 7 a 48 dias $(5,12)$. A paciente descrita neste relato difere da literatura por ter sido diagnosticada mais tardiamente e por ter feito uso da insulina por tempo mais prolongado. Entretanto, é possível que ela já manifestasse sintomatologia de DM muito antes de ter procurado assistência médica, uma vez que foi diagnosticada em cetoacidose diabética, uma manifestação de hiperglicemia crônica. Em relação ao uso prolongado da insulinoterapia, desde aproximadamente seis meses após seu início, a menor passou a fazer uso apenas da insulina regular, para tratar ocasionais hiperglicemias, sugerindo retorno a um estado de controle glicêmico muito anterior à suspensão definitiva de qualquer insulinoterapia seis 
meses depois. Além disso, os níveis de hemoglobina glicada sempre estiveram normais durante o tratamento, mesmo usando doses pequenas de insulina, e têm-se mantido normais, 15 meses após a suspensão do tratamento.

Pui e cols. (13) avaliaram fatores de risco para a ocorrência de hiperglicemia em 421 pacientes com LLA em uso de asparaginase. Idade maior que 10 anos, obesidade e síndrome de Down foram significativamente associadas com a complicação e, quando combinados, esses fatores exerciam efeito sinérgico (13). Sexo, raça, contagem leucocitária e esquema de uso da droga não foram associados ao desenvolvimento de diabetes (13). Em nossa paciente, apenas a idade se destacou como possível fator de risco para a complicação, uma vez que, além de não ter síndrome de Down, apresentava índice de massa corpórea dentro da normalidade.

\section{CONCLUSÃO}

A ocorrência de diabetes melito em pacientes tratados com L-asparaginase é uma complicação rara, porém importante, da terapia de pacientes com neoplasias hematopoiéticas malignas. O DM é insulinopênico e de caráter transitório, resolvendo após a suspensão da L-asparaginase. Desta forma, oncologistas e endocrinologistas devem estar atentos para a possibilidade do surgimento desta complicação, monitorando níveis glicêmicos em todos os pacientes que fazem uso de L-asparaginase. A investigação para DM tipo 1A, DM insulino-resistente induzido por corticoterapia e DM secundário à pancreatite tóxica é mandatória. O tratamento com insulinoterapia deve ser acompanhado de perto devido à natureza transitória desse tipo de diabetes.

\section{AGRADECIMENTOS}

Esse trabalho foi parcialmente financiado pela FAPESB (Fundação de Amparo à Pesquisa do Estado da Bahia).

\section{REFERÊNCIAS}

1. Krogh CME. Compendium of pharmaceuticals and specialties. 27th ed. Ottawa: Canadian Pharmaceutical Association, 1992. p. 590

2. Clavell LA, Gelber RD, Cohen HJ, Hitchcock-Bryan S, Cassady $\mathrm{JR}$, Tarbell NJ, et al. Four-agent induction and intensive asparaginase therapy for treatment of childhood acute lymphoblastic leukemia. N Engl J Med 1986;315:657-63.
3. Pui $\mathrm{CH}$, Evans WE. Treatment of acute lymphoblastic leukemia. N Engl J Med 2006;354:166-78.

4. Zubrod CG. The clinical toxicities of L-asparaginase. Pediatrics 1970;45:555-9.

5. Charan VD, Desai N, Singh AP, Choudhry VP. Diabetes mellitus and pancreatitis as a complication of L-asparaginase therapy. Indian Pediatr 1993;30:809-10.

6. Howard SC, Pui C. Endocrine complications in pediatric patients with acute lymphoblastic leukemia. Blood Rev 2002; $16: 225-43$.

7. Gillette PC, Hill LL, Starling KA, Fernbach DJ. Transient diabetes mellitus secondary to L-asparaginase therapy in acute leukemia. J Pediatr 1972;81:109-11.

8. Faletta JM, Steuber CP, Hayes JW, Starling KA, Hill LL. Nonketotic hyperglycemia due to prednisone (NSC - 10023) following ketotic hyperglycemia due to L-asparaginase (NSC 109229) plus prednisone. Cancer Treat Rep 1972;56:781-2.

9. Whitecar JP, Bodey GP, Hill CS Jr, Samaan NA. Effect of Lasparaginase on carbohydrate metabolism. Metabolism 1970;19(8):581-6.

10. Lavine RL, Brodsky I, Garofano CD, Rose LI. The effect of $E$. coli L-asparaginase on oral glucose tolerance and insulin release in man. Diabetologia 1978;15:113-6.

11. Carpentieri U, Balch MT. Hyperglycemia associated with the therapeutic use of L-asparaginase: possible role of insulin receptors. J Pediatr 1978;93(5):775-8.

12. Turner GR, Marks JF, Buchanan GR. Relative hyperglucagonemia in L-asparaginase-and-prednisone-induced glucose intolerance in management of acute lymphocytic leukemia. Clin Pediatr 1983:22:363-7.

13. Pui CH, Burghen GA, Bowman WP, Aur RJA. Risk factors for hyperglycemia in children with leukemia receiving L-asparaginase and prednisone. J Pediatr 1981;99(1):46-50.

14. Bächman LA. Post-transplant diabetes mellitus: the last 10 years with tracolimus. Nephrol Dial Transplant 2004:19(suppl. 6):vi13-6.

15. Duval M, Suciu S, Ferster A, Rialland X, Nelken B, Lutz $P$, et al. Comparison of Escherichia coli-asparaginase with Erwinia-asparaginase in the treatment of childhood lymphoid malignancies: results of a randomized European Organization for Research and Treatment of Cancer - Children's Leukemia Group phase 3 trial. Blood 2002;99:2734-9.

16. Ridgway D, Neerhout RC, Bleyer A. Attenuation of asparaginase-induced hyperglycemia after substitution of the Erwinia carotovora for the Escherichia coli enzyme preparation. Cancer 1989;63:561-3.

17. Eisenbarth GS, Polonsky K, Buse JB. Type 1 diabetes mellitus. In: Larsen PR, Kronenberg HM, Melmed S, Polonsky KS (eds). Williams Textbook of Endocrinology. $10^{\text {th }}$ ed. Philadelphia: Saunders, 2003. pp. 1485-508.

Endereço para correspondência:

Crésio Alves

Rua Plínio Moscoso 222, apto. 601

40157-190 Salvador, BA

E-mail: cresio.alves@uol.com.br 